\title{
Numerical simulation of droplets, bubbles and waves: state of the art
}

\author{
Daniel Fuster ${ }^{1,2}$, Gilou Agbaglah ${ }^{1,2}$, Christophe Josserand $^{1,2}$, \\ Stéphane Popinet ${ }^{3}$ and Stéphane Zaleski ${ }^{1,2} \ddagger$ \\ ${ }^{1}$ Univ Paris 06, UMR 7190, Institut Jean Le Rond dAlembert, F-75005 Paris, France. \\ ${ }^{2}$ CNRS, UMR 7190, Institut Jean Le Rond dAlembert, F-75005 Paris, France. \\ ${ }^{3}$ National Institute of Water and Atmospheric research, Wellington, New Zealand
}

\begin{abstract}
This work present current advances in the numerical simulation of twophase flows using a VOF method, balanced-force surface tension and quad/octree adaptive mesh refinement. The simulations of the atomization of a liquid sheet, the capillary retraction of a liquid sheet and two- and three-dimensional wave breaking all for air/water systems, are used to show the potential of the numerical techniques. New simulations of atomization processes for air/water conditions are allowing to investigate the processes leading to the appearance of instabilities in the primary atomization zone in real conditions. For the retracting liquid sheet, the new simulations show that two different regimes can be encountered as a function of the Ohnesorge number. For large values, a laminar flow is encountered inside the rim and a steady state is reached after a quick transient state. For small values, a turbulent flow is generated inside the rim which is responsible of large oscillations in the rim size and neck thickness. The breaking wave case study demonstrates the orders-of-magnitude efficiency gains of the adaptive mesh refinement method.
\end{abstract}

Keywords: two-phase flows, atomization, liquid sheet, Gerris, VOF, numerical simulation, breaking wave

\section{Introduction}

The physics and particularly the dynamics of two-phase flow have grown into a major scientific domain with crucial fundamental issues as well as many practical and industrial applications. Fields of interests range from drop impact phenomena involved in the study of rain on soils or foliage, ink-jet printing, and combustion chambers [46, 40]. In particular atomization processes [37] play an important role in combustion studies. Twophase flow is also prevalent in geophysical contexts such as river motion and meandering or ocean dynamics [33]. The dynamics of interfaces is rich and complex, with many mathematical and technical issues that need to be resolved to account for the specific role of these interfaces (a surface in $3 \mathrm{D}$, a line in 2D) separating two domains with different physical properties. Recently, the experimental access to high-speed imaging

$\ddagger$ Corresponding author: stephane.zaleski@upmc.fr 
and the systematic use of self-similar approaches and matched asymptotics methods (see for instance [7]) have greatly improved our general understanding of multiphase flows. These developments and the recent improvements in numerical techniques have also made it possible to tackle problems which were still inaccessible a few years ago, such as 3D impacts or sprays dynamics. All these aspects have tremendously increased the interest for the ever more complex and realistic two-phase flow simulations that are now feasible. The motivation of this paper is thus to present recent achievements in two-phase flow study based on up-to-date numerical methods. The numerical techniques used here combine two classical methods, namely, the Volume-of-Fluid method (VOF) that allows a good treatment of the interface, with the Adaptive Mesh Refinement (AMR), which optimize the numerical calculations as discussed earlier in [39, 17]. In this paper, after a brief review of important recent developments in numerical methods, we illustrate what is now achievable through three typical two-phase flow studies in air-water conditions.

\section{Numerical method}

Most multiphase flow problems for practical applications exhibit all or several of the following characteristics: high surface-tension, low viscosity, high density ratios, complex and evolving interface topologies, spatial scales ranging over several orders-ofmagnitude. In this context, an ideal numerical method for the solution of the two-phase Navier-Stokes equations with surface tension would have the following properties:

- robust representation of evolving, topologically complex interfaces,

- accurate representation of surface tension which requires accurate estimates of interface normal and curvature,

- robust and accurate handling of large density and viscosity ratios,

- efficient representation of evolving flow features of widely different characteristic spatial scales.

While distinct numerical methods have one or several of these properties, arguably no method published to date possesses all of them together. Methods using an implicit representation of the interface such as Volume-of-Fluid (VOF) [21] or Level-Set (LS) [23] can robustly and efficiently represent evolving, topologically complex interfaces but generally suffer from an inaccurate representation of surface tension $[18,8]$. Methods using an explicit representation of the interface such as Arbitrary-Lagrangian-Eulerian (ALE) discretisations [9, 3] or Front-Tracking (FT) [18, 22] can provide an accurate representation of surface tension but have difficulty dealing with complex and evolving interface topologies. Finally dynamic mesh-adaptive methods can deal efficiently with phenomena involving a wide range of spatial scales but their implementation to date has been limited to a small subset of the methods referred to above.

Recently one of us proposed new extensions of these methods which allow to combine their respective strengths and thus come closer to the ideal numerical method 
described above [17]. The basis for the numerical scheme is the incompressible adaptive Navier-Stokes solver Gerris $[15,16]$ which uses an octree for spatial discretisation. This type of discretisation was shown to lead to efficient adaptivity for single-phase flows (the cost of adapting the mesh at every timestep is generally less than $5 \%$ of the total computing effort). This approach is different from the more classical block-structured adaptive schemes of [1] in that each discretisation element can be refined independently from the others. This fine-scale adaptivity was shown in [17] to be very relevant to flows with interfaces where it is often desirable to refine on and along interfaces only. While doing this is possible with block-AMR techniques, the finite size of AMR blocks leads to significant increases in total mesh sizes.

A challenge is then to extend classical interface-tracking techniques to these adaptive meshes. A simple approach is to restrict adaptivity only to the bulk of each phase. This guarantees that the interface between phases never changes resolution and leads to a trivial implementation of existing methods [10]. This comes at the cost of never being able to refine along the interface. As was shown in [17] and as is further illustrated in the present article, this restriction can lead to a dramatic decrease of the efficiency of adaptivity for realistic problems. To lift this restriction, Popinet [17] proposed to extend the VOF method to adaptive octree grids. The technique rests on the creation of virtual regular Cartesian stencils, reconstructed dynamically from the underlying octree discretisation. Using the algorithmic properties of octrees, simple and efficient algorithms can be designed to achieve this. The resulting technique allows full adaptivity along the interface while keeping all the other advantages of the VOF method: arbitrary interface topology and topological changes, good mass conservation properties, sharp interface description. Figures 1 and 17 illustrate VOF-reconstructed interfaces with a spatial resolution varying along the interface obtained with the Gerris solver.

An oft-mentioned drawback of VOF methods is the difficulty of obtaining accurate values for useful geometrical properties such as interface normal directions or curvature. This is often contrasted with the relative simplicity with which these values can be obtained with e.g. Level-Set techniques. A recent study by François et al. [8] casts doubt on this simplistic interpretation however and shows that obtaining accurate secondorder curvature estimates in the Level-Set (LS) method is not trivial. This article also compares LS-derived curvature estimates with a "rediscovered" height-function (HF) curvature estimation using a VOF fraction field. It shows that the HF scheme gives a second-order accurate estimate of curvature from VOF volume-fraction fields while being simple to implement on regular Cartesian grids. While this is true for well-resolved interfaces, a problem occurs when the interface curvature becomes comparable to the mesh size. In this limit the standard HF method becomes inconsistent. The problem is addressed in Gerris by switching to a novel generalised HF method for low resolutions which ensures consistent second-order convergence of the curvature estimates across all resolutions [17]. Gerris further generalises the HF method to octree grids by using the virtual regular Cartesian stencils method mentioned previously. 


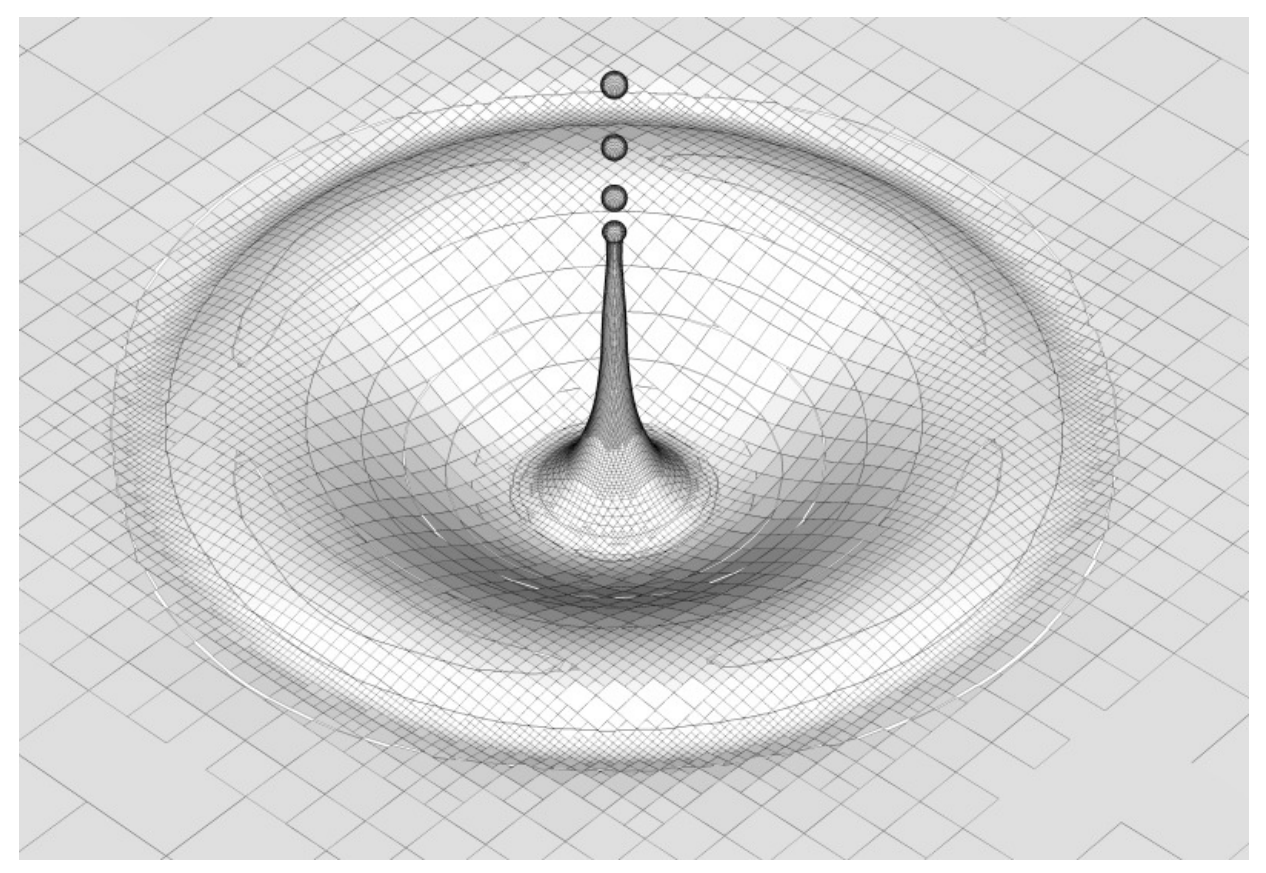

Figure 1. Jet created by the collapse under surface tension of an air bubble just below the surface of still water. The interface is represented using the VOF-reconstructed fragments in each cell. The resolution varies along the interface according to local interface curvature. The finest scales (droplets and jet) are 128 times more refined than the coarsest scales (flat surface in the corners of the picture). This three-dimensional, two-phase, air-water simulation can be compared with the axisymmetric, free-surface (i.e. single phase) simulations of [6]. Accurate surface tension, robustness for large density ratios and changes of topology as well as adaptivity are all necessary to obtain these results.

While computing accurate curvature estimates is an essential step to obtaining an accurate surface tension formulation, it is not sufficient. The inaccuracy of surface tension formulations has been the traditional weak point of methods using an implicit representation of the interface (such as VOF or levelset). A classical simple test case is that of a bubble or droplet at rest in a surrounding fluid. In this case the exact solution is given by Laplace's law stating that - for a constant surface tension coefficient - the pressure jump across the interface exactly balances the surface tension force, resulting in a stationary, spherical interface [18]. Up until recently all surface tension formulations coupled with an implicit interface representation failed this simple test case and produced non-equilibrium solutions with so-called "spurious" or "parasitic" currents of varying amplitude depending on parameters such as: viscosity, density ratio, spatial and temporal resolution etc... At worst, these parasitic currents can be strong enough to dominate the solution. This is of particular concern for practical applications which often fall in the parameter range where parasitic currents are strong. Although there has been a vast number of papers trying to address the problem in the past ten years, the method implemented in Gerris and published in [17] is the first which 
Table 1. Amplitude of spurious currents for a selection of methods published in the literature. The spatial resolution is given in number of grid points per droplet diameter. The amplitude of spurious currents is given in non-dimensional form as: $\max |\mathbf{u}| \sqrt{D / \sigma}$ with $D$ the droplet diameter and $\sigma$ the surface tension coefficient. La is the Laplace number: $\sigma \rho D / \mu^{2}$ with $\mu$ the viscosity and $\rho$ the density of the liquid.

\begin{tabular}{|c|c|c|c|c|c|c|c|c|c|}
\hline \multirow[b]{2}{*}{ Method } & \multirow[b]{2}{*}{ Year } & \multirow[b]{2}{*}{ La } & \multicolumn{7}{|c|}{ Spatial resolution } \\
\hline & & & 6.4 & 12.8 & 16 & 24 & 25.6 & 32 & 40 \\
\hline VOF-CSS[11] & 1995 & 12,000 & & & & -0.5 & & & \\
\hline FT-Markers[18] & 1999 & 12,000 & $5 \mathrm{e}-3$ & $9 \mathrm{e}-4$ & & & $1 \mathrm{e}-4$ & & \\
\hline GFM[13] & 2000 & 728,000 & & $3 e-4$ & & & & & \\
\hline VOF-PROST[19] & 2002 & 0.357 & & & & $3.75 \mathrm{e}-5$ & & $2.19 \mathrm{e}-5$ & $1.6 \mathrm{e}-5$ \\
\hline FT-Markers[22] & 2005 & 12,000 & & & & & $2 \mathrm{e}-4$ & & \\
\hline Level-Set-VOF[24] & 2007 & 12,000 & $8 \mathrm{e}-2$ & $5 \mathrm{e}-4$ & & & $6 \mathrm{e}-6$ & & \\
\hline $\mathrm{DG}[2]$ & 2008 & 12,000 & & & $1 \mathrm{e}-2$ & & & & \\
\hline Level-Set[12] & 2008 & 12,000 & $5 \mathrm{e}-4$ & $1.6 \mathrm{e}-4$ & & & $3.7 \mathrm{e}-5$ & & \\
\hline VOF-HF $[17]$ & 2009 & $\leq 12,000$ & & & & $-<10$ & & & \\
\hline
\end{tabular}

demonstrates recovery of an exact equilibrium solution for this simple test case (see Table 1). Furthermore this exact solution was also shown to be obtained irrespective of spatial resolution or physical parameters. The method stresses the importance of the concept of "balanced-force" continuum formulation introduced in [19] and couples it with the generalised HF method to obtain exact equilibrium solutions.

The overall scheme is simple to implement, only requires a single VOF interface representation (in contrast to coupled methods such as VOF/markers and VOF/Levelset), works on octree grids allowing adaptivity along the interface, conserves mass and provides accurate surface tension solutions for a range of test cases.

Table 1 summarises the progresses made in tackling the problem of "parasitic currents" around a stationary spherical droplet with surface tension. The initial VOF Continuum-Surface-Stress implementation of [11] had very strong spurious currents independently of spatial resolution. This problem was addressed in [18] by switching to a Front-Tracking (markers connected by third-order splines) representation of the interface coupled with a "surface-tension corrected" pressure gradient. While spurious currents were drastically reduced using this approach and showed convergence with resolution, the marker technique was only two-dimensional and could not deal with topology changes. The PROST method of [19] was the first VOF method with low spurious currents we are aware of. It introduced the important concept of "balancedforce" surface tension representation in the context of continuum-surface-force methods. The paraboloid-fitting method used to compute curvature from volume fractions was very computationally expensive however. Most other methods published since were refinements of these earlier methods and showed similar amplitudes of spurious currents. The VOF, height-function, balanced-force method implemented in Gerris is the first 
technique which demonstrated convergence to exact equilibrium (to within machine precision) irrespective of Ohnesorge number and spatial resolution [17].

Finally large density ratios have also proven difficult to handle using methods with an implicit representation of the interface. For such methods it is tempting and often necessary to smooth the jumps in physical properties (including density) occuring at the interface. While this leads to simple finite-difference discretisations of the (now continuous) partial differential equations describing two-phase flows, the resulting numerical approximations can lead to physically-inconsistent results. Several schemes have been proposed to try to address some of these problems: for example both the "pressure gradient correction" scheme [18] and the "ghost-fluid method" [13] introduce better (and similar) discretisations of the pressure jump due to surface tension forces but do not address the discontinuity in fluid momentum and/or viscous stresses due to jumps in density and viscosity. While mass conservation has always been a key advantage of VOF methods (compared to e.g. Level-Set methods), momentum conservation has generally received less attention. Many implementations thus discretise the non-conservative form of the variable-density Navier-Stokes equations which has the advantage of using the continuous velocity field as primary variable, rather than the discontinuous momentum field. The density field and velocity field are then advected using different schemes (e.g. VOF for density and centered/upwind scheme for velocity) which can lead to anomalous numerical momentum diffusion across the interface. To address this problem Rudman [20] proposed to advect momentum using the same VOF scheme as for density so that momentum conservation was recovered. This scheme relies on a sharp interface representation and is not compatible with smoothing. We intend to implement this scheme in Gerris but at present we use a non-momentum-conserving velocity/density formulation.

A related problem for large density ratios is that the Poisson problem for the pressure - derived from the incompressibility condition - becomes stiffer as the density jump increases. This can again be somewhat minimised by smoothing the interface but as discussed previously this is not a satisfactory solution (although this is often done in practice). Gerris uses the multigrid Poisson solver presented in [15] for singlephase flows which fits nicely with the octree discretisation. This solver generally works well also for two-phase flows with large density ratios (as demonstrated in this article), however its performance (in terms of convergence speed) depends - as for any geometric multigrid solver - on the consistency of the representation of domain (i.e. interface) topology on successively coarser grids. For complex flows this consistency is not guaranteed and this can lead to important performance penalties. A range of recent linear solver techniques could be used to address this problem including: algebraic multigrid, multigrid-accelerated GMRES, Krylov-subspace methods etc... and we plan to explore this issue further in the future.

To study the accuracy of the surface-tension representation in the case of large density ratios, we considered the case of $2 \mathrm{D}$ oscillations of a liquid drop floating in a lighter fluid. The density ratio is $1 / 1000$. No explicit viscosity is added so that any 
damping of the oscillations is due to numerical diffusion only. The droplet diameter is 0.4 and it oscillates in a $2 \times 2$ square domain. The initial shape of the droplet is given by:

$$
r(\theta)=r_{0}+\epsilon \cos (n \theta)
$$

The theoretical small-amplitude inviscid oscillation frequency is then [14]:

$$
\omega_{n}^{2}=\frac{\left(n^{3}-n\right) \sigma}{\left(\rho_{d}+\rho_{e}\right) r_{0}^{3}}
$$

This test case is similar to that used by Torres and Brackbill [25] and Hermann [12] and we compare the results obtained using Gerris with the results presented in both of these papers. A comparison between the theoretical and numerical values of the frequency is given in Figure 2 for $n=2$ and $\epsilon=0.05$.

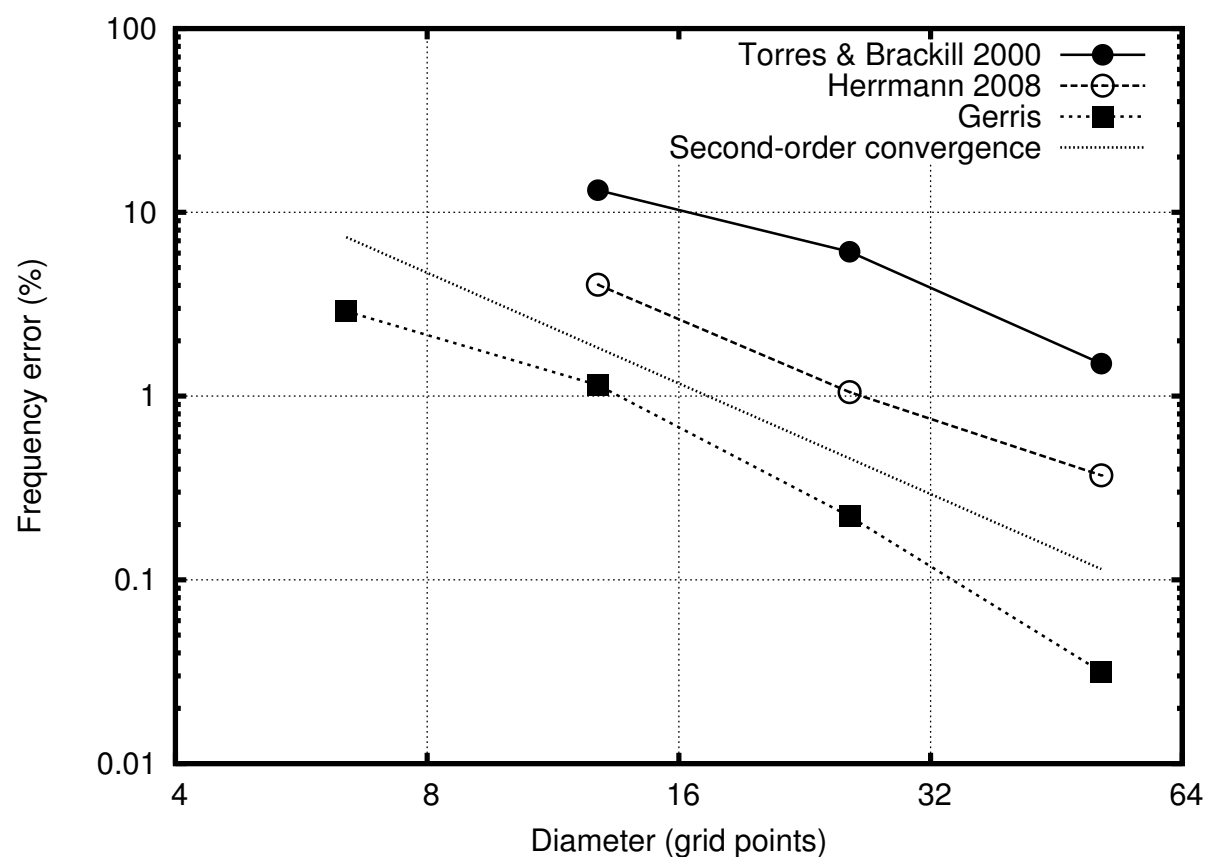

Figure 2. Convergence of the error between the computed and theoretical frequency for the mode 2 oscillations of a liquid droplet.

Note that the results of Torres \& Brackbill and Herrmann were obtained while using a small explicit viscosity and for density ratios of $1 / 100$. This test case was initially selected by Torres \& Brackbill to demonstrate the importance of accurate surface tension representation. Given the low explicit viscosity, any spurious currents due to inaccurate surface tension will not be damped and will strongly affect the solution. The accuracy of Gerris in this respect is confirmed by this test case. Another measure of the accuracy of the solution is the estimation of the numerical damping induced by the various discretisation errors of the numerical scheme. The amount of numerical damping can be estimated by computing an equivalent viscosity. With viscosity, kinetic energy is 
expected to decrease as:

$$
\exp \left(-C \nu / D^{2} t\right)
$$

where $C$ is a dimensionless constant (set empirically to 30), $\nu$ the viscosity and $D$ the droplet diameter. Using curve fitting the damping coefficient $b=C \nu / D^{2}$ can be estimated (solid lines on Figure 3.a). An equivalent Laplace number can then be computed as:

$$
\mathrm{La} \equiv \frac{\sigma D}{\rho \nu^{2}}=\frac{\sigma C^{2}}{\rho b^{2} D^{3}}
$$

The numerical viscosity and thus the equivalent Laplace number depend on spatial resolution as illustrated in Figure 3.b. The kinetic energy is seen to behave in a physically-consistent manner at all resolutions. High Laplace numbers can be reached at high resolution which confirms that the overall stability of the method is not due to an excess of numerical viscosity.

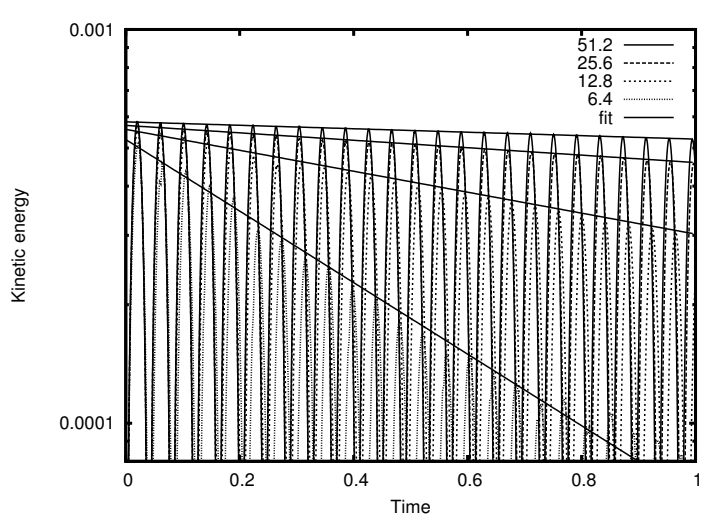

(a)

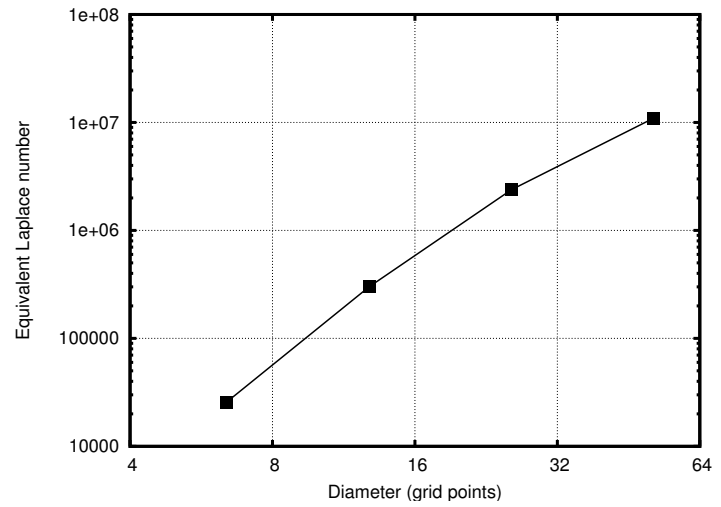

(b)

Figure 3. (a) Evolution of the total kinetic energy for an oscillating droplet for the spatial resolutions given in the legend (in grid points per diametre). (b) Equivalent Laplace numbers.

In what follows we demonstrate how the combination of adaptivity, accurate surface tension and overall robustness of the method leads to novel applications by focusing on three problems: two-dimensional atomisation, retraction of liquid sheets and small-scale air-water wave breaking.

\section{Atomization}

Atomization processes are of significant interest in various industrial and research fields. However, our understanding of the physical processes leading to the break-up of liquid masses is still far from complete. Although different phenomena have been separately studied in the literature, the complexity of the analysis of the whole processes has prevented investigators from obtaining a complete understanding of all the processes 
underlying atomization.

Nowadays, numerical simulations has become a major avenue of investigation for atomization processes, alongside theory and experimentation. Thanks to the recent developments introduced earlier in this paper, it is now possible to perform simulations with a high level of accuracy in conditions very close to those of atomization experiments.

Generally, three different parts of the processes have been investigated separately, namely, the flow inside the injector, the primary atomization zone near the injector and the secondary atomization zone further downstream. The flow inside the injector establishes the input boundary conditions at the chamber. In the primary atomization zone, the liquid jet which is injected into the chamber is exposed to the high-velocity gas flow. The perturbations induced at the interface quickly grow downstream becoming nonlinear and producing long ligaments which eventually break into droplets. Finally, in the secondary atomization zone, large fragments of the central jet zone and droplets created upstream in the primary atomization region may be further broken into droplets or coalesce and the undisturbed jet core cannot be distinguished anymore.

Different spatial scales appear across the domain. In some parts, as near the injector, a fine mesh resolution is required to capture the correct wave amplification, the ligament formation or the ligament break-up. In other parts, as in the secondary atomization zone, fine mesh resolution is required to capture the whole range of droplet sizes generated there or carried from upstream. However, with current computational resources, such a degree of resolution cannot be applied to the entire domain and Adaptive Mesh Refinement (AMR) techniques are required in order to concentrate the computational effort just in those zones where it is needed.

When the whole process is investigated, one of the main issues is the presence of strong interactions between different parts of the system: the flow upstream, that is inside the injector nozzle, determines the parameters of the atomization at the entrance of the chamber; such as the intensity of turbulence introduced in the system or the size of the boundary layers. The flow in the secondary atomization zone can create some vortices and droplets. These can be sent back upstream by recirculating flow and influence the flow in the primary atomization zone. The present work aims at capturing all the interactions between the different parts of the system. Although some assumptions and simplifications are still required, it is shown that current advances in the numerical simulation of atomization processes are enough to capture important information and to recover quantitative experimental measurements for real air/water systems. 
Table 2. Simulation conditions for the analysis of atomization processes.

\begin{tabular}{ccccc} 
& $U(\mathrm{~m} / \mathrm{s})$ & $\rho\left(\mathrm{kg} / \mathrm{m}^{3}\right)$ & $\mu(\mathrm{Pa} \cdot \mathrm{s})$ & $\sigma(\mathrm{N} / \mathrm{m})$ \\
\hline gas & 20 & 1.2 & $1.7 \cdot 10^{-5}$ & 0.069 \\
liquid & 0.173 & 1000 & $10^{-3}$ &
\end{tabular}

\subsection{Simulation of atomization processes in conditions close to experiments}

In this section, the effect of the injector in the primary atomization zone is investigated. In order to perform simulations with large resolution in big domains, the twodimensional problem is considered.

3.1.1. Simulation set-up Conditions contained in Table 2 are used in this work to show the potential of the code to handle air/water simulations.

These conditions, which define the characteristic dimensionless parameters contained in Table 3, correspond to the experimental investigation of air/water atomization processes at large momentum ratios $M=\rho_{l} U_{l}^{2} /\left(\rho_{g} U_{g}^{2}\right)$ reported in ref. [30].

Table 3. Representative dimensionless numbers for the conditions given in Table 2, where $\mu$ is the viscosity, $\delta$ is the thickness of the boundary layer at the entrance, $\rho$ is the density and $\sigma$ is the surface tension.

\begin{tabular}{cccccc}
\hline$m$ & $r$ & $R e_{l}$ & $R e_{g}^{*}$ & $W e_{g}^{*}$ & $M$ \\
$\mu_{g} / \mu_{l}$ & $\rho_{g} / \rho_{l}$ & $\rho_{l} U_{l} H_{l} / \mu_{l}$ & $\rho_{g} U_{g} \delta_{g} / \mu_{g}$ & $\rho_{g}\left(U_{g}\right)^{2} \delta_{g} / \sigma$ & $\frac{\rho_{g} U_{g}^{2}}{\rho_{l} U_{l}^{2}}$ \\
\hline 0.017 & $1.2 \cdot 10^{-3}$ & 1730 & 714 & 3.52 & 16
\end{tabular}

The simulation set-up is depicted in Figure 4. Although the main goal is to analyze the characteristic frequencies and flow patterns near the injector, it has been found that both parameters can be much influenced by the domain boundaries if the domain is not large enough. If the output boundary condition is imposed before the jet is definitely broken into droplets, some wave reflection can be induced at the outflow boundaries and they significantly modify the characteristic frequencies observed some distance downstream. In order to avoid this effect, a large domain of $16 \mathrm{~cm}$ is considered. The liquid and gas height of the sheet at the entrance are, for both phases: $H_{g}=H_{l}=1 \mathrm{~cm}$. At the entrance, two boundary layers are introduced to reproduce the velocity profiles near the solid walls $\left(\delta_{l}=\delta_{g}=179 \mu \mathrm{m}\right)$. Between the liquid and gas jets, a separator plate with a minimum thickness of $150 \mu \mathrm{m}$ and a slope (or opening half-angle) of $\beta=7^{\circ}$ is included where non-slip boundary conditions at the walls are imposed (the separator plate can be seen more clearly in the zoomed view of Figure 7). The inclusion of the separator plate inside the simulation domain is important to capture some of the effects 


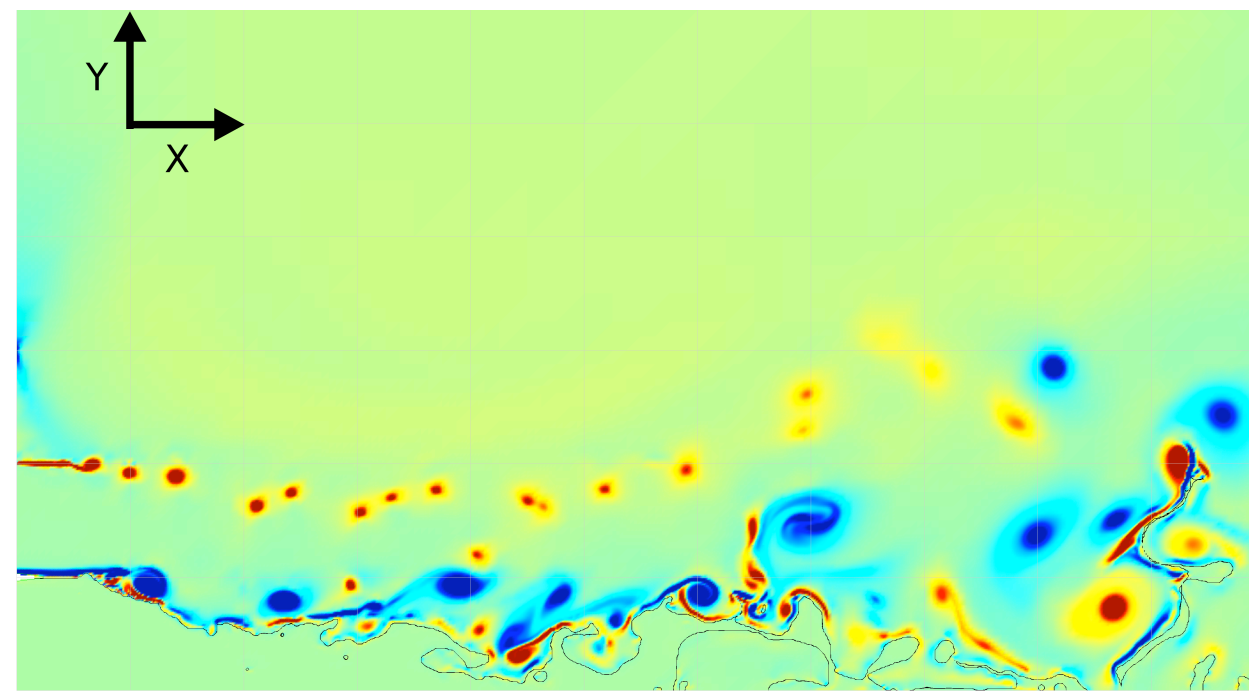

Figure 4. Vorticity field and interface representation when a quasi steady state is reached. Domain size: $11 \times 6 \mathrm{~cm}$.

of the injector geometry on the flow (e.g. to capture the effect of the thickness of the separator plate $e$ and the opening half-angle $\beta$ ).

Finally, a standard outflow boundary condition (zero normal derivative of normal velocity and zero pressure) is imposed at the right boundary of the domain whereas slip boundary conditions are set at all other boundaries.

Regarding the mesh, an automatic octree refinement based on two different criteria has been used: the gradient of the VOF color function and the norm of the vorticity. The first criteria allows us to control the degree of accuracy with which the interface is captured. The second one, controls the level of resolution achieved to capture the turbulent vortices. Both criteria are also space-dependent in order to concentrate the computational effort in the zone near the injector. In particular, the maximum spatial resolution in the different parts of the domain is:

- $9.76 \mu \mathrm{m}$ for $x<2 \mathrm{~cm}$ and $y<3 \mathrm{~cm}$,

- $39 \mu \mathrm{m}$ for $2<x<3 \mathrm{~cm}$ and $y<3 \mathrm{~cm}$,

- $156 \mu \mathrm{m}$ for $x>3 \mathrm{~cm}$ and $y<3 \mathrm{~cm}$.

- For $y>3 \mathrm{~cm}$, , the interface is refined with cells of $156 \mu \mathrm{m}$ if some liquid reaches this zone. If no liquid is present, a coarse mesh is used to dampen the vortices entering in this zone.

In addition, the grid near the solid walls is maximally refined $(9.76 \mu \mathrm{m})$ in order to maximize the accuracy of the simulation near the separator and to capture the generation of instabilities by the trailing edge of the separator plate. 
We would like to stress the importance of octree refinement (Figure 5).

- On one hand, it has allowed us to consider a domain where the boundaries are relatively far from the injector without a prohibitive increase of the number of cells. For distances further than $2 \mathrm{~cm}$ from the injector, the mesh is progressively coarsened. Thus, although the accuracy of the results in this zone is not as good as near the injector, the influence of the boundary conditions on the observed frequencies is negligible.

- On the other hand, very refined meshes can be used near the injector (of the order of 10 microns) which allows us to perform high-accuracy simulations of the flow in this zone.

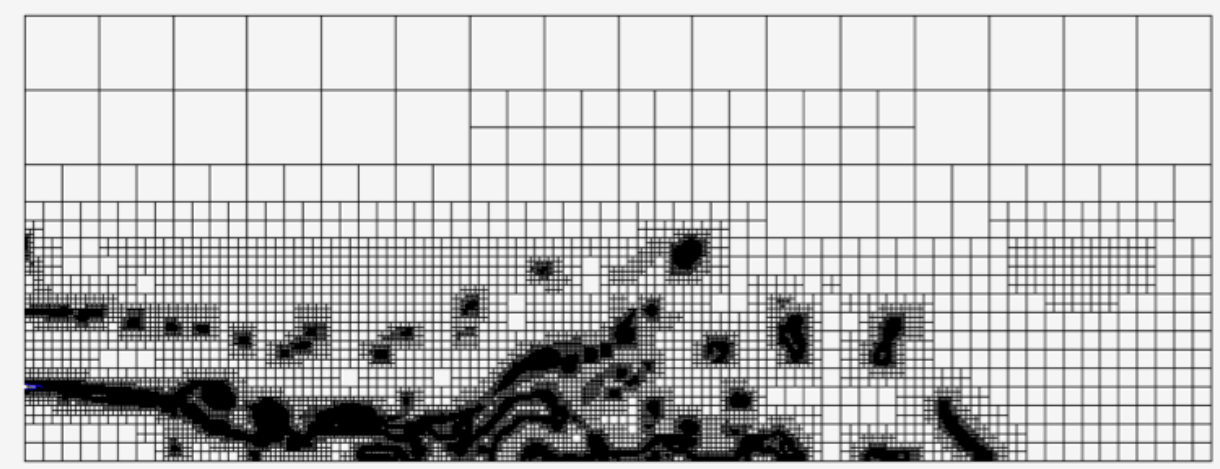

Figure 5. Simulation domain and one example of a typical mesh distribution.

3.1.2. Analysis of the simulation results The new simulations allow to capture various flow patterns in the different parts of the domain (Figure 4). At the trailing edge of the separator plate, the liquid is exposed to the high-velocity gas sheet and the exchange of momentum between the gas and liquid jets accelerates the liquid. The amplitude of the waves generated at the interface is significant even immediately downstream of the trailing edge.

Qualitatively, we can observe the amplification of the instabilities induced near the injector as well as the generation of ligaments which are finally broken into droplets. The presence of the liquid potential cone is also clearly distinguished. In Figure 6, the superposition of the graph of the interface during $0.2 \mathrm{~s}$ reveals that the length of the potential cone is between 3 and $4 \mathrm{~cm}$, which agrees with the experimental correlation found in $[30]$ :

$$
L_{\text {cone }}=12 M^{-1 / 2} H_{L}=3 \mathrm{~cm}
$$

Focusing the analysis in the zone near the injector, we can observe waves of relatively large amplitude immediately behind the injector (of the order of the boundary 


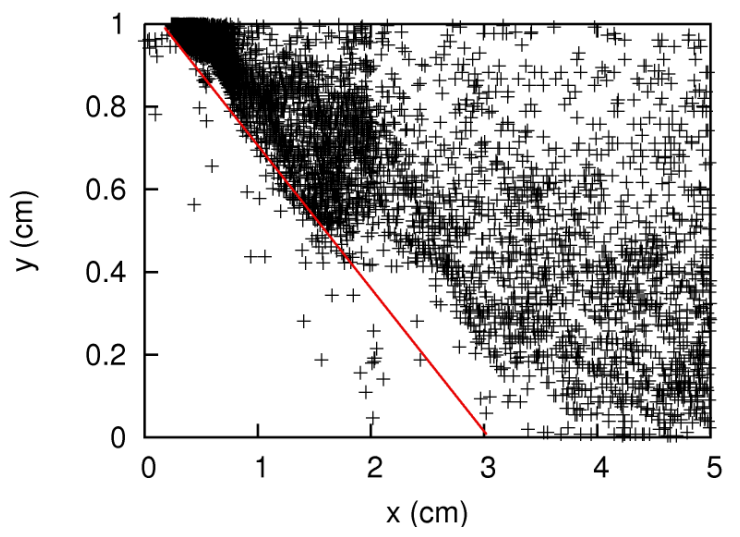

Figure 6. Dots: Projection of the position of the interface during 0.2s. Line: Slope of the potential cone extracted from the experimental correlation.

layer thickness and the separator plate thickness). The generation of these waves turns out to be a periodic process summarized in the sequence of images depicted in Figure 7 and described below:
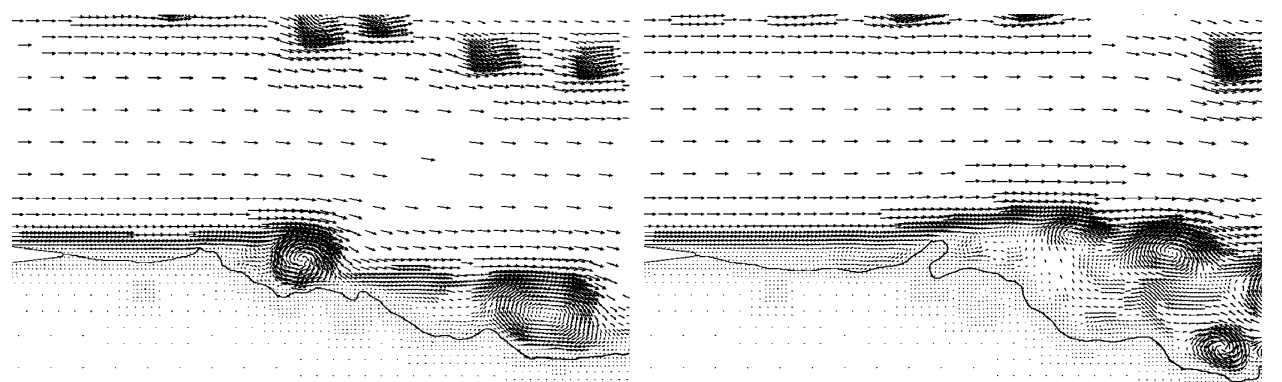

A

B

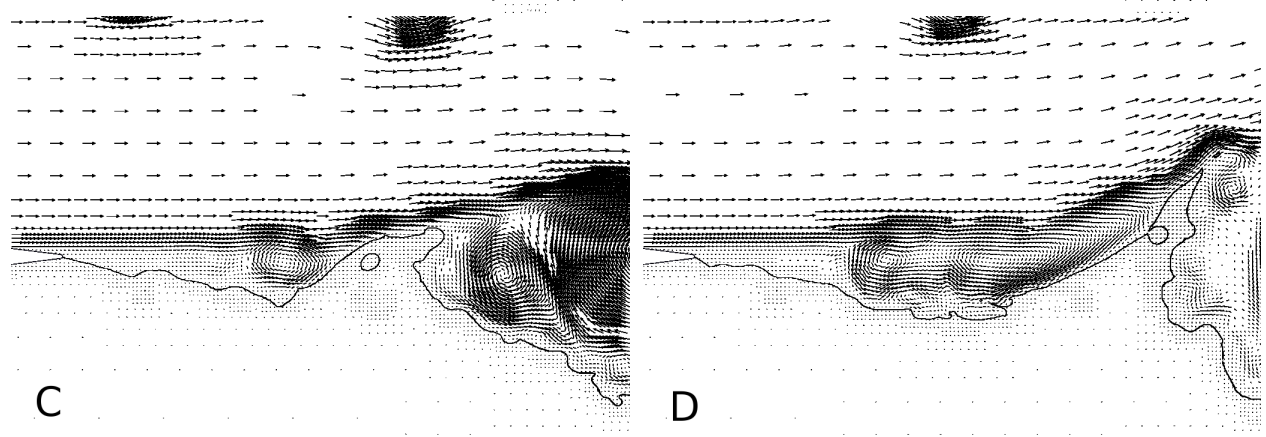

Figure 7. Zoom of the flow near the separator plate. Sequence of images of the generation, growth and propagation of the perturbation induced near the injector. Time lag between images: $5 \mu \mathrm{s}$.

After one wave has been advected downstream, the liquid level in the injector surroundings is approximately constant and it is sustained for a relatively long distance 
compared with the thickness of the separator plate. At this moment, some perturbations are induced at the interface due to the turbulence generated in the gas phase near the interface. These disturbances can have an amplitude as large as the thickness of the separator plate. When it occurs, the wave amplifies and once the crest of the wave goes beyond the gas boundary layer, a sudden acceleration and amplification is produced. Due to the large amplitude of the wave propagating downstream, the mass conservation makes the liquid level around the injector decrease. The volume which was initially occupied by the liquid is now filled with a recirculating gas flow. During these moments, the effective thickness of the gas boundary layer is much larger than the amplitude of the disturbances induced at the interface (Figure 8). The decrease of the liquid level is
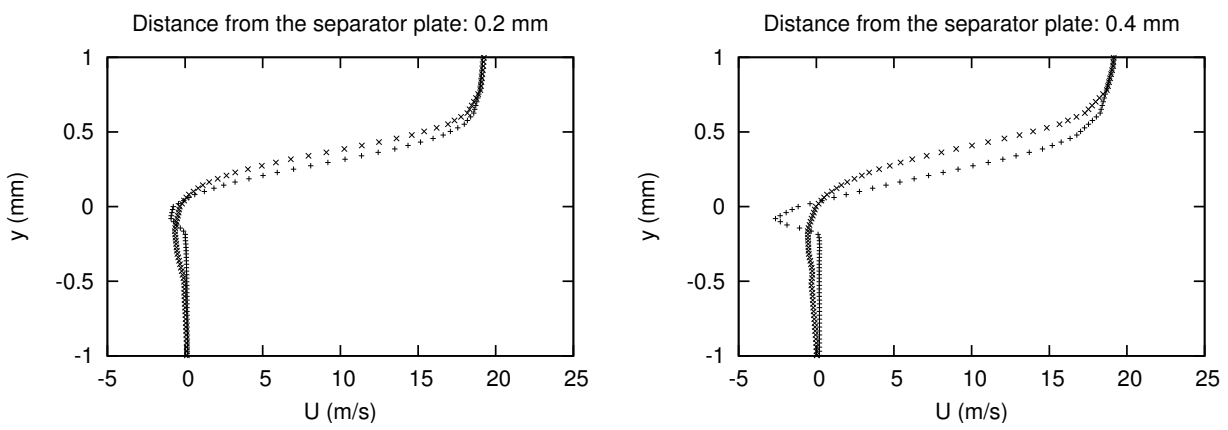

Figure 8. Two examples of the instantaneous profiles of the $x$ velocity component when the interface is at the level of the injector $(+$ dots) and when the liquid level has decreased due to the amplification and propagation of a large wave downstream (x dots).

large enough to avoid any wave reaching the gas bulk. In addition, the large gas vortices produced in the gas boundary layer make the waves propagate in both directions, up and downstream. At this moment, the disturbances induced at the interface are not in contact with the gas jet and are not amplified. It is not until the liquid level near the injector is recovered that the next wave of large amplitude appears.

When a big wave is generated, some ligaments appear which eventually are broken in relative large droplets (see Figure 9). This process governs the appearance of droplets near the injector and it will be discussed in more detail in the next section.

Then, we can conclude that the current simulations have been shown to capture a realistic behavior of the atomization processes for air/water systems at large momentum ratios. This result is remarkable if we take into account the shortage of numerical simulations for this kind of systems due to the different numerical issues related with the large density and viscosity ratios encountered at the interface. The presence of the separator plate has made it possible to introduce correct boundary conditions at the entrance, and thanks to AMR, large domains can be simulated making it possible to diminish the influence of the boundary conditions on the dynamics near the separator 


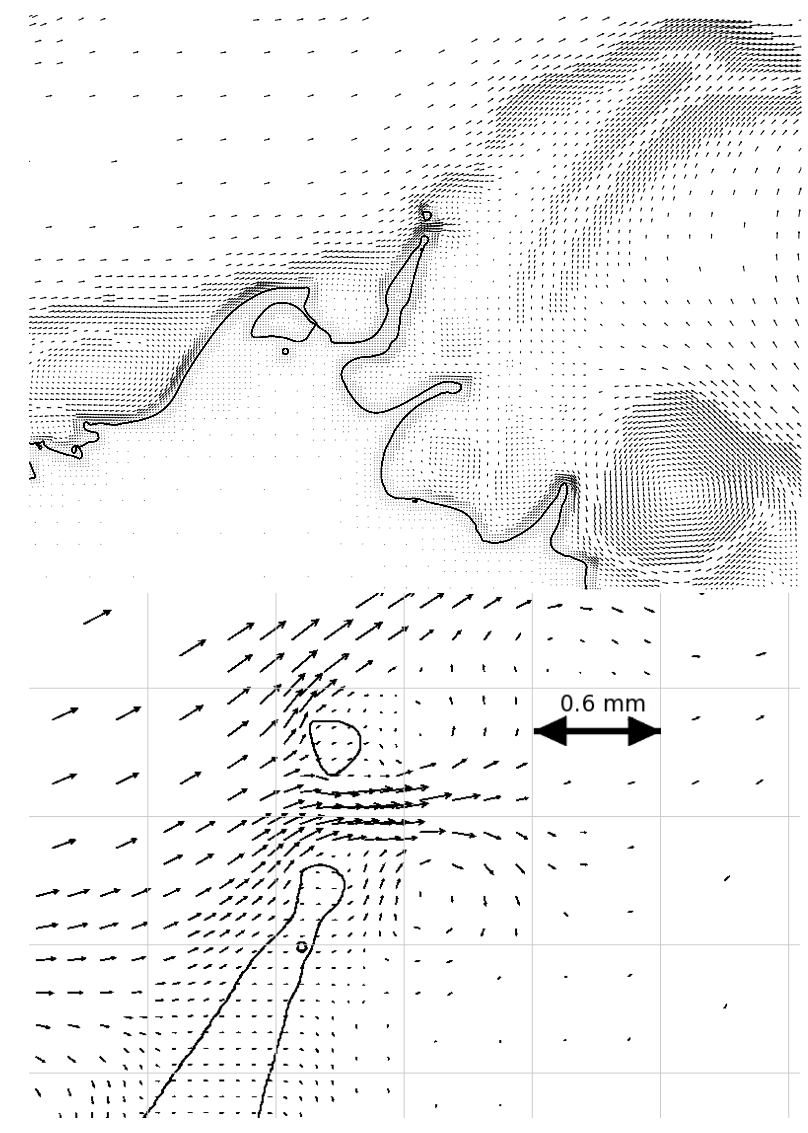

Figure 9. Generation of a single drop from a ligament in the primary atomization zone. For the sake of clarity, the velocity vectors are just depicted at level of refinement $8(78 \mu \mathrm{m})$. Top: global view. Bottom: a zoom near the topmost part of the interface.

plate.

\section{Capillary retraction of a liquid sheet}

\subsection{Introduction}

During the atomization, drops may be formed by several distinct mechanisms. A general understanding of these processes is still lacking and is at the heart of many fundamental studies on atomization: Kelvin-Helmholtz instabilities, jet and filament break-up, and liquid sheet deformation. In particular, the destabilization of a liquid sheet is known to detach small droplets in situations such as the crown splash illustrated by the iconic photographs of Edgerton[36].

A particular feature of liquid sheet dynamics is that its free ends retract because of the surface tension, thus forming a rim that usually grows with time and is often unstable. As explained and shown by Taylor [45] and Culick [34], the retraction dynamics of a homogeneous liquid sheet initially at rest reaches a constant speed, the so-called Taylor-Culick velocity $v_{T C}=\sqrt{2 \sigma} \rho e$, where $\sigma$ is the surface tension, $\rho$ the 
Table 4. Characteristic parameters used to write the rest of variables in dimensionless form

$$
\begin{array}{ccc}
L_{c}(\mathrm{~m}) & U_{c}(\mathrm{~m} / \mathrm{s}) & \rho_{c}\left(\mathrm{~kg} / \mathrm{m}^{3}\right) \\
\hline 5 \cdot 10^{-3} & 1 & 10^{3}
\end{array}
$$

liquid density and $e$ the thickness of the liquid sheet. This velocity comes from the momentum balance between the liquid entering into the rim with the surface tension. A full description of the dynamics of the liquid sheet requires three-dimensional analysis and extensive numerical simulations and is beyond the scope of the present paper. Here, we will instead focus on the two-dimensional retraction dynamics. Although we know that the 2D flows thus obtained may be unstable in three dimensions [38], the properties of the $2 \mathrm{D}$ retraction are remarkable and a better understanding of these dynamics is needed to improve our understanding of rim instability even in 3D. Rich and complex behaviors have already been observed in 2D, such as capillary waves and a pinching of the film that becomes more pronounced with surface tension [44] or viscous-capillarybalance retraction speed $[31,35,4]$. Here we will show in particular that the $2 \mathrm{D}$ film retraction does not pinch the liquid film and that it becomes unstable at low Ohnesorge numbers.

\subsection{Numerical simulation}

Our aim is thus to numerically investigate the motion and stability of the edge of a liquid sheet in 2D. We consider a two-dimensional flow and we try to be as close as possible to the typical flows of an air/water system. The capillary retraction is controlled by the density and viscosity ratios, and by the Ohnesorge number $\left(\mathrm{Oh}=\mu /\left(\rho \sigma e_{0}\right)^{1 / 2}\right)$ where $e_{0}$ is the initial homogeneous thickness of the sheet. Notice that $\mathrm{Oh}=\sqrt{l_{v c} / e_{0}}$ where

$l_{v c}=\mu^{2} /(\rho \sigma)$ is the viscous-capillary length. For water, notice that $l_{v c} \simeq 14 \cdot 10^{-9} \mathrm{~m}$, a particularly small length. For a sheet with $e_{0} \simeq 50 \cdot 10^{-6} \mathrm{~m}$ ( five times the finest grid size used in the simulation of Section 3.1.2), we thus have $\mathrm{Oh} \simeq 0.017$. We perform the study in the reference frame where the film edge is at rest to allow large computation times. In this framework, both the liquid and the air are entering into the calculation domain (at the left boundary, see Figure 10) at the Taylor-Culick velocity $v_{T C}$.

The physical parameters used in the simulations to obtain a dimensionless system are summarized in Table 4.

We use a computational domain of size $4 \times 1$ in dimensionless units. The initial mesh has $4 \times 2^{7} \times 2^{7}$ cells. The mesh is adapted based on two criteria, the interface position and the magnitude of the vorticity field, in order to maintain a good resolution around the interface as well as wherever the vorticity is large.

Finally, a Dirichlet boundary condition is imposed on the velocity field on the left 
boundary where the gas and the liquid are penetrating while an outlet condition is taken on the right-hand side. The density ratio is 850 and viscosity ratio is 50 which corresponds to an air/water system. In our dimensionless units, the initial length of the sheet is $18 e_{0}$ and the unit-square subdomain corresponds to $20 e_{0}$ (see Figure 10). We introduce the liquid capillary time $\tau=\sqrt{\rho_{L} e_{0}^{3} / \sigma}$ which allows to define the dimensionless time $t^{*}=\frac{t}{\tau}$.

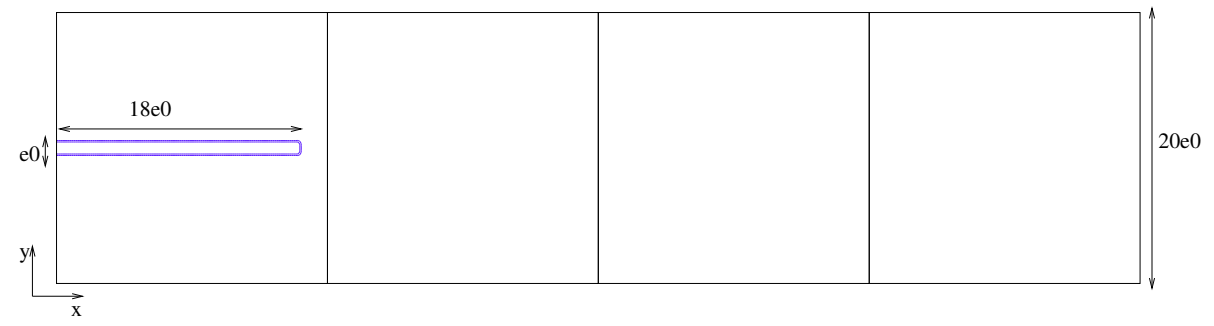

Figure 10. Spatial domain of the retracting sheet.The domain consists of $4 \times 1$ square subdomains. The liquid sheet is surrounded by a gas with a density 850 times smaller

\subsection{Results}

Figure 11 shows snapshots of the liquid sheet evolution at eight different times and for four different Ohnesorge numbers ranging from 0.005 to 0.1 . We observe that after a short transient (a few units of times), the rims almost stay centered on the same position as time evolves. This demonstrates that the rim recedes at the Taylor-Culick speed $v_{T C}$.

This figure illustrates also an instability mechanism in the retraction dynamics for small Ohnesorge numbers. Indeed, for high Ohnesorge numbers, (0.1 and 0.055 in Figure 11), viscous effects are important and we observe a monotonic increase of the rim (see Figure 11 (a) and (b)). On the other hand, as the Ohnesorge number decreases, the evolution of the rim is significantly different. As shown in Figure 11 (c) and (d), the rim is not circular anymore but elongated in the $x$ direction and oscillations of the rim are observed.

A neck is formed at the front of the rim. This neck is followed in the sheet by a stationary capillary wave whose amplitude is exponentially decreasing in space (L. Gordillo, private communication and G. Agbaglah, L. Gordillo, C. Josserand and S. Zaleski, to be submitted.) The stationary capillary waves structure along the liquid sheet is more pronounced for low Ohnesorge, but it is important to notice that no breakup of the liquid sheet has been observed in the numerics, in contrast to the prediction of finite-time singular pinch-off [44]. The appearance of a finite-time singularity is wellknown for 3D capillary dynamics such as liquid jet pinch-off for instance [7]. Here, however, the surface tension cannot enhance the finite time singularity in 2D and the neck is dominated by the balance between capillarity, inertia and viscous stress. 


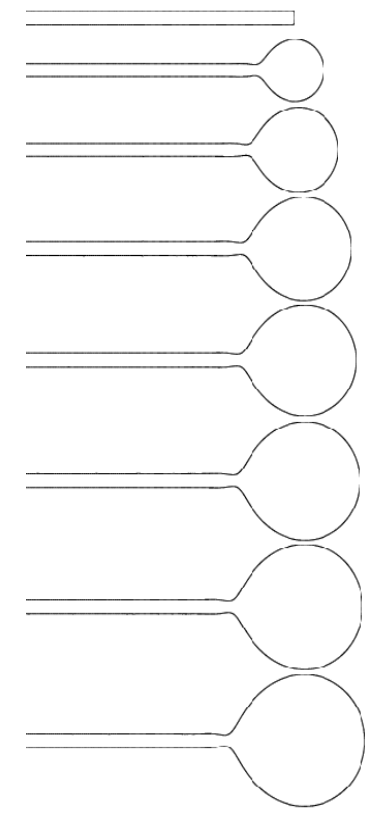

(a)

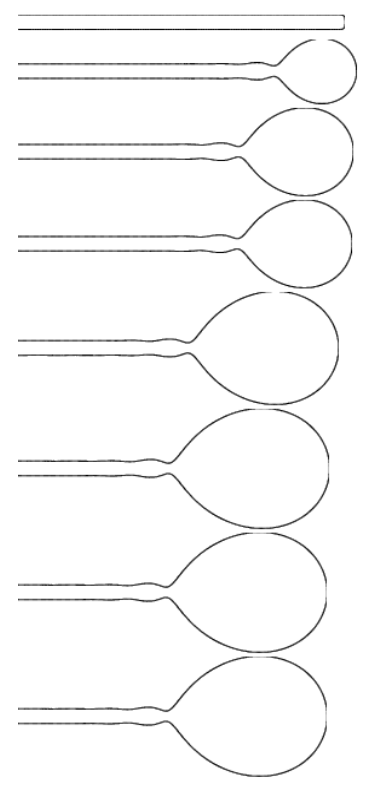

(b)

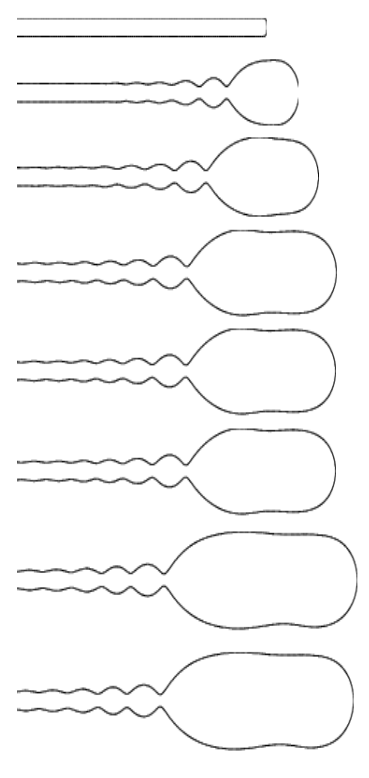

(c)

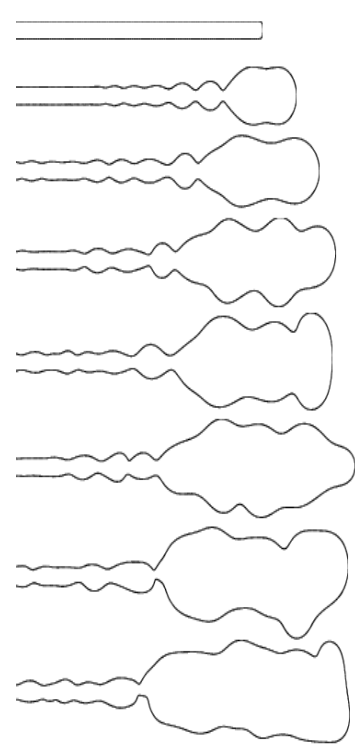

(d)

Figure 11. Evolution of the sheet interface at $t^{*}=$ $0.00,5.29,10.58,15.87,21.17,31.75,42.33,52.92$ (from top to bottom) for four different Ohnesorge numbers: 0.1 (a), 0.055 (b), 0.01 (c), 0.005 (d). A rim is formed at the edge of the free end of the sheet and a neck appears just behind the rim.

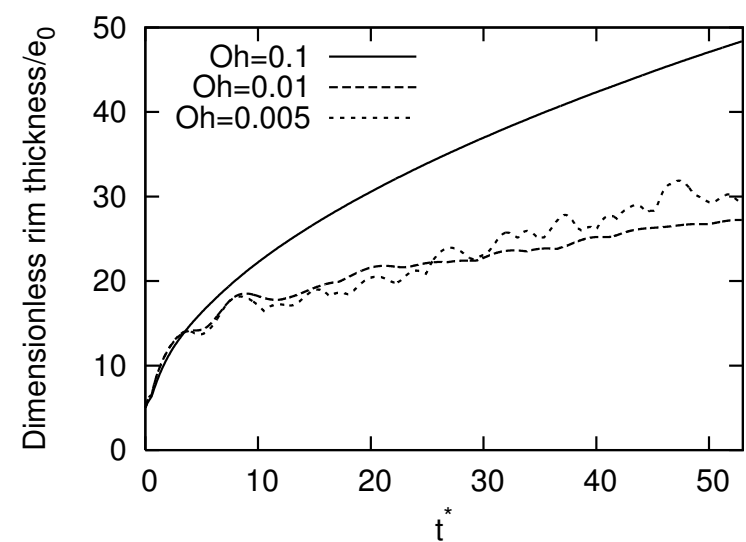

(a)

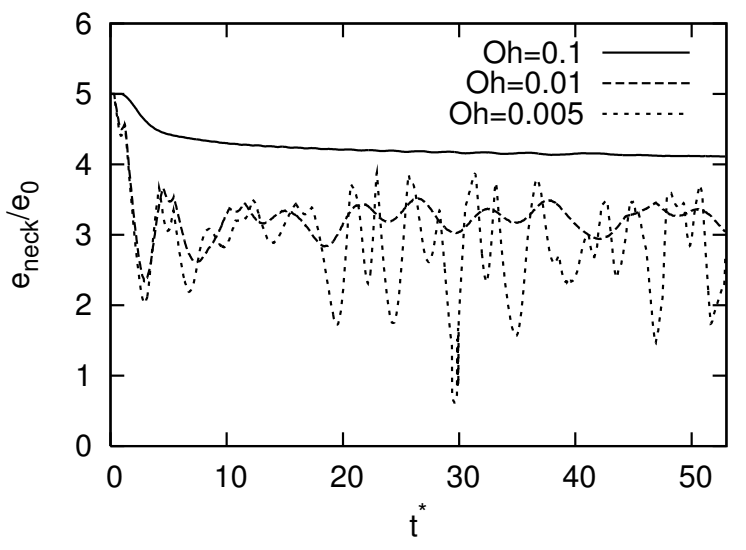

(b)

Figure 12. (a) Evolution of the size of the rim for $O h=0.1, O h=0.01$ and $O h=0.005$. (b) Evolution of the neck thickness for $O h=0.1, O h=0.01$ and $O h=0.005$. The sheet is stable for $O h=0.1$ and becomes unstable for $O h$ smaller than 0.01

Figure 12, where the rim and neck thickness are plotted for several Ohnesorge numbers, also illustrates the change of behavior in the retraction dynamics. While the 


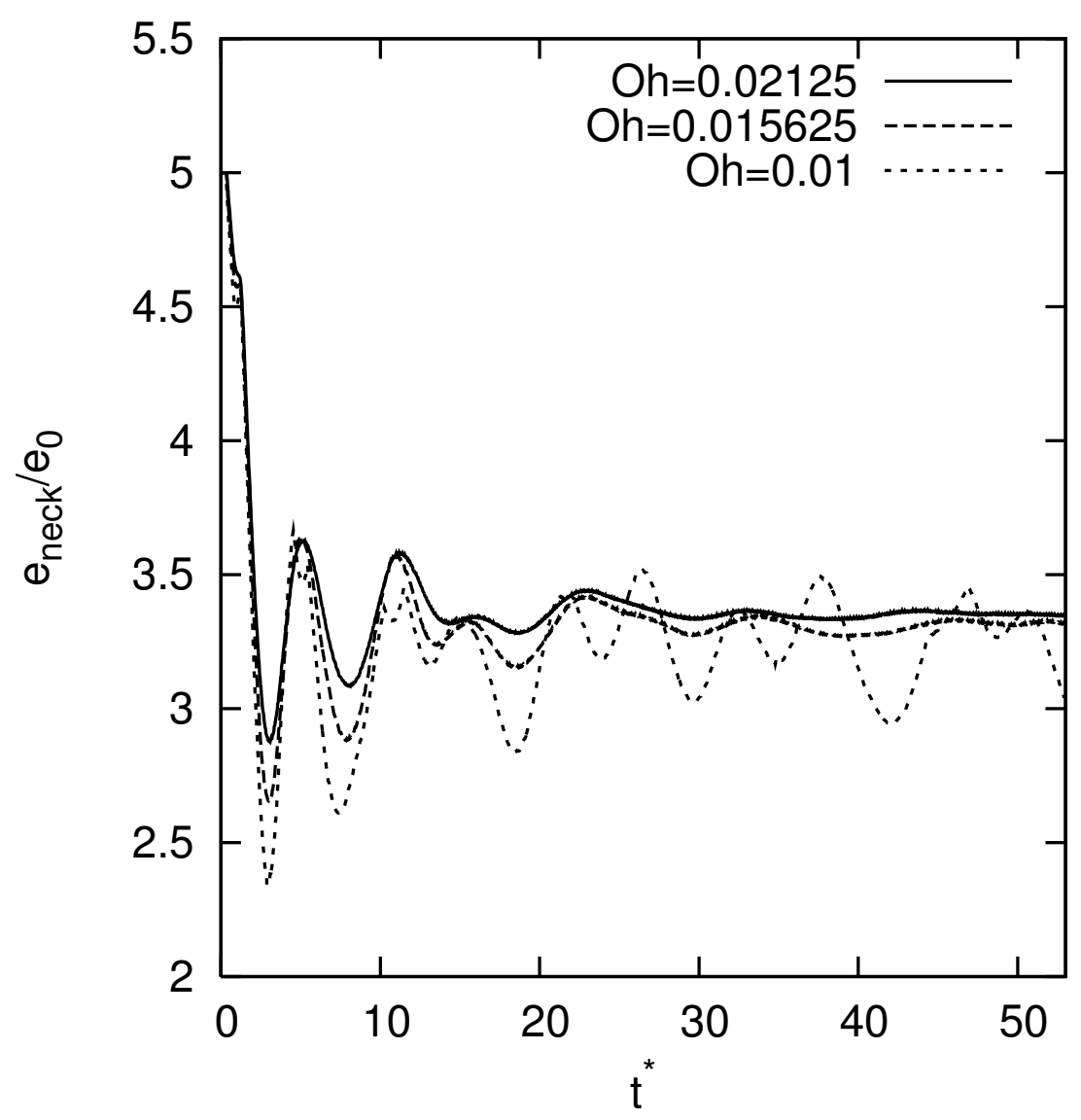

Figure 13. Evolution of the neck thickness for different $O h$. A transition regime is encountered for $O h$ between 0.016 and 0.01

large Ohnesorge number (0.1 here) shows the expected square-root-of-time regime[41], the elongation of the rim is obvious for smaller Oh number $(0.01$ and 0.005 in the figure). Similarly, Fig 12 (b) shows a smooth convergence of the neck thickness for the highest Ohnesorge number while strong and irregular oscillations of this thickness can be observed for the smaller Ohnesorge numbers.

Finally, Figure 14 shows that the instability of the edge of the sheet is in fact related to the presence of vortices nucleated by the dynamics. For low Ohnesorge numbers, the vorticity, created by the neck and capillary wave structures is advected inside the rim and makes it oscillate. Such highly vortical flows can indeed destabilize the rim (see [44]). For large Ohnesorge numbers, the vorticity remains concentrated around the neck and no instability can develop. This suggests that the instability can be seen as a particular case of a Kelvin-Helmholtz instability, due to the shear between the gas and the retracting liquid sheet.

From our set of numerical simulations, we deduce that this instability develops for Ohnesorge numbers smaller than $0.013 \pm 0.003$. For an air-water system, this corresponds to a film thickness approximately larger than 100 microns. 


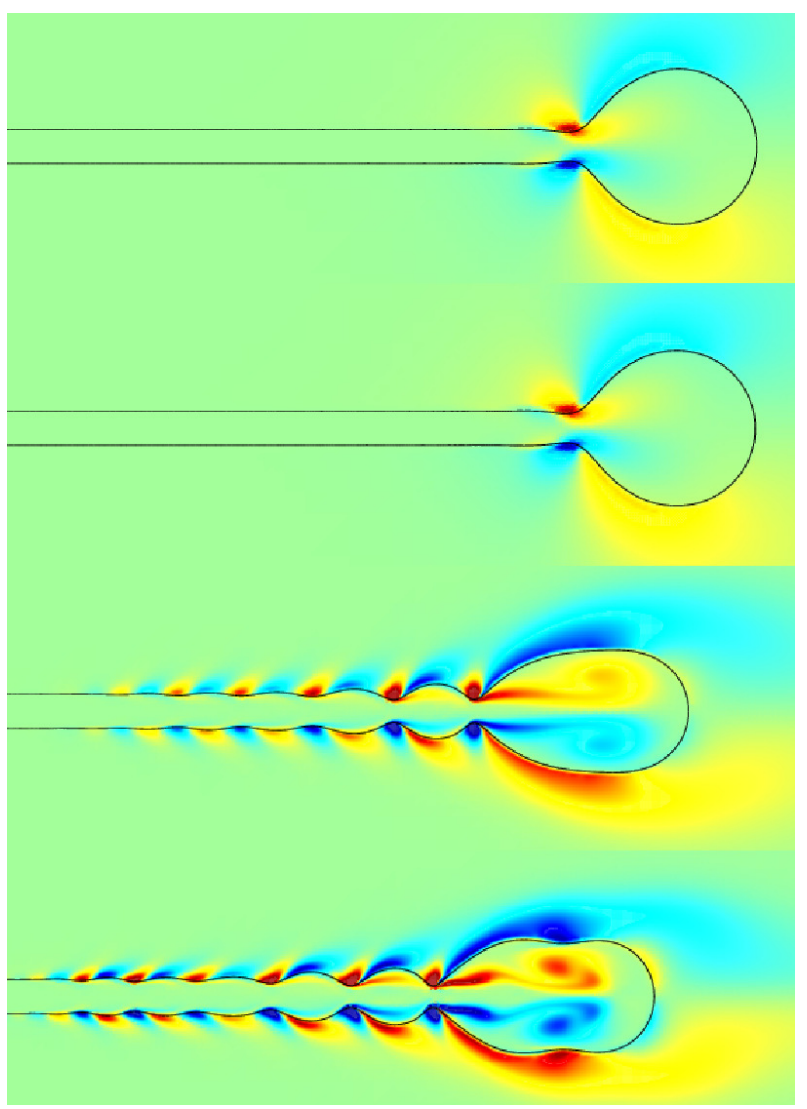

Figure 14. Vorticity field at $t^{*}=5.29$ for different Ohnesorge numbers (From top to bottom: $0.1,0.055,0.01,0.005)$. A pair of vortices appears inside the rim only for $O h=0.01$ and $O h=0.005$

\section{Wave breaking}

Aside from the combustion-engine injectors discussed earlier, atomization of liquidgas interfaces is also typical of the breaking of steep air-water waves. This is an important problem in geophysics since the dynamics of water waves controls a number of fundamental processes including the transfer of soluble gases between the atmosphere and the ocean and the formation of atmospheric aerosols. Depending on wave steepness various regimes can be distinguished: linear waves, weakly non-linear waves, stable non-linear waves, spilling waves, plunging breakers etc... Also - while the water phase largely controls the transfer of slightly soluble gases for weakly non-linear waves - the air phase must be taken into account when dealing with steep spilling or breaking waves. Small-scale waves are predominant at the ocean surface and may be responsible for an important fraction of the global ocean-atmosphere fluxes. At these scales surface tension plays an important role.

From a numerical analysis perspective two main trends can be distinguished: 
(i) Potential flow theory in conjunction with boundary-integral methods has been successfully applied to the study of the long-time evolution of large-scale water waves and their interactions [5].

(ii) Direct numerical simulation (DNS) techniques initially developed in the context of channel-flow turbulence studies have been extended to flows beneath a fixed or weakly-deformed free surface [27].

Both of these approaches have important limitations. Potential flow techniques cannot describe the boundary layers controlling gas exchange while existing DNS methods do not include the non-linear deformations of the interface and/or are limited to a single phase $[27,26]$. Further advances could be made by using recently developed methods for the direct simulation of two-phase interfacial flows. While a few studies using these techniques have been published, they are often restricted in scope due to two main limitations: inaccuracies of the numerical methods (particularly regarding momentum conservation and surface tension representation) and prohibitive computational cost at high spatial resolutions (particularly in three dimensions). Both these limitations are addressed in the recent developments discussed earlier. This section illustrates the potential of these new techniques for the study of steep non-linear air-water waves.

An example of the results obtained for the evolution of a small-amplitude viscous Stokes wave is given in Figure 15. Non-dimensional times $t^{\prime}=t \sqrt{g / \lambda}$ with $\lambda$ the wavelength, are 1.32, 2.65, 3.97 and 5.30 from top to bottom. Surface tension is included which leads to the formation of capillary waves riding in front of the gravity wave. No external forcing is added and the amplitude of the wave slowly decreases due to viscous dissipation. Both the thin viscous boundary layer in the water phase and the thicker boundary layer in the gas phase are evident with strong vorticity generation associated with the high curvatures of capillary waves. These results are similar (for the water phase) to those obtained by Tsai et al [26] using their single-phase free-surface solver.

As with other implicit front-capturing methods, the interface topology is not restricted: air entrainment and spray formation can thus be modelled directly provided high-enough resolution. Figure 16 illustrates preliminary results for the simulation of a three-dimensional unstable steep Stokes wave. To illustrate three-dimensional breaking the initial third-order Stokes wave steepness parameter $a k$ is varied from 0.35 to 0.45 in the direction transverse to the wave motion. The boundary conditions are periodic in the direction of wave motion and symmetrical in the transverse direction. A characteristic plunging breaker is formed at $t^{\prime}=2$, then leads to the trapping of a large air "tube" and the formation of a secondary plunging sheet at $t^{\prime}=2.5$. The edge of this sheet is rounded due to capillary retraction. Eventually holes form immediately behind the sheet's edge and grow due to capillary retraction, leading to the formation of cylindrical "fingers" which are themselves unstable due to Plateau-Rayleigh instability. At $t^{\prime}=2.8$ the sheet has impacted with the front of the wave and further atomization occurs as the droplets themselves impact on the interface.

As in the previous simulation, the octree mesh is adapted at every time step 
according to the local magnitude of vorticity and interface curvature. Figure 17 illustrates a detail of the variable-resolution VOF representation of the interface. The maximum resolution allowed is set to $\Delta / \lambda=1 / 1024$. For the Reynolds and Bond numbers of 40,000 and 33,333 chosen, this guarantees that both the smallest viscous and capillary scales are resolved. This is confirmed by the observation (Figure 17 for example) that most droplets and bubbles have sizes significantly larger than the mesh size.

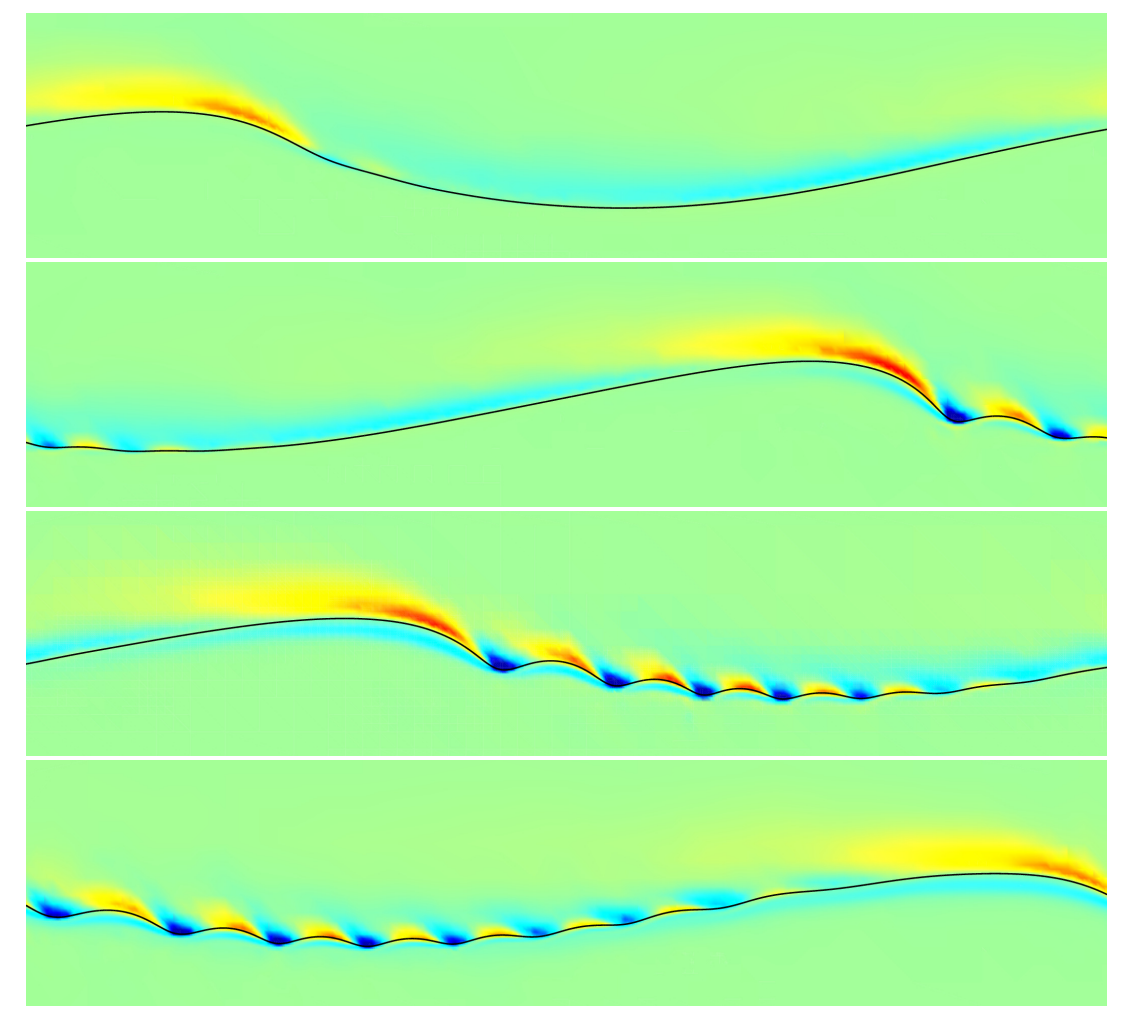

Figure 15. Evolution of a small-amplitude third-order Stokes wave with surface tension. $a k=0.28, \operatorname{Re}=\sqrt{g \lambda^{3}} / \nu=40000$, Bo $=\rho \lambda^{2} g / \sigma=333$, air/water density and viscosity ratios. Colour represents vorticity. The wave is travelling from left to right.

Adaptivity is crucial for obtaining the results presented here. Figure 18 illustrates the evolution with time of the total number of grid points. The initial wave is smooth and can be resolved accurately with a limited number of elements. As the plunger forms, local interface curvature increases and requires higher resolution. Plunger impact at $t^{\prime}=2.1$ creates other highly-curved structures and finally sheet atomisation at $t^{\prime}=2.5$ further increases the resolution requirements. The final number of grid points of $\approx 1.4$ million can be compared to the $\approx 1$ billion grid points which would be required if a regular Cartesian grid was used. Even a method using adaptivity but with a constant interface resolution would require about 50 times more grid points. 


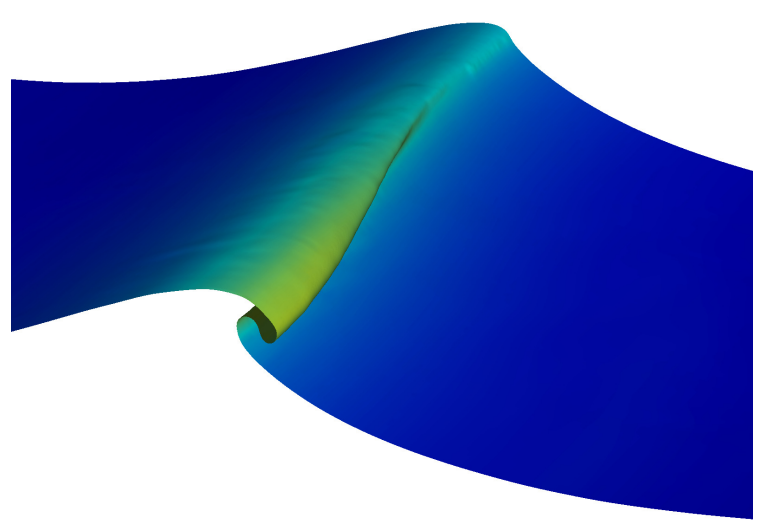

$t^{\prime}=2$

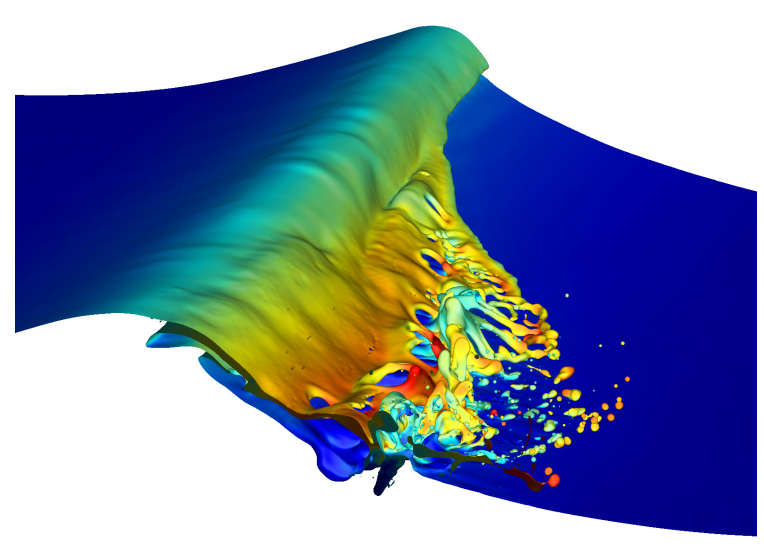

$t^{\prime}=2.8$

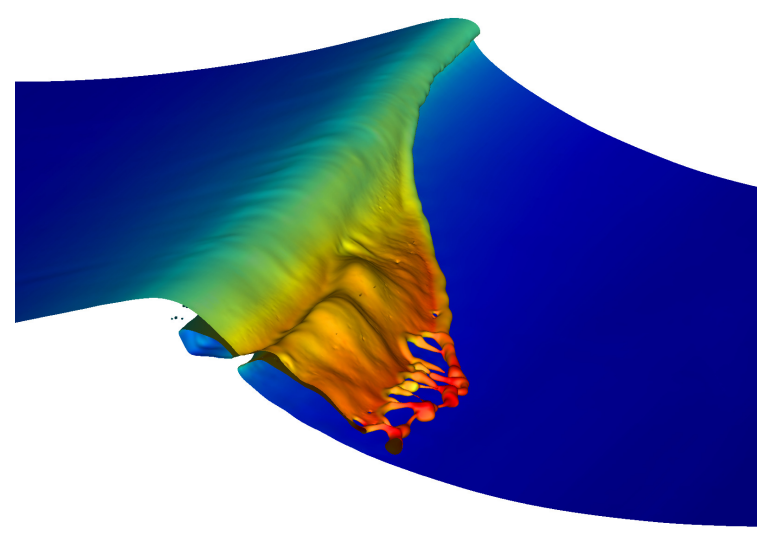

$t^{\prime}=2.5$

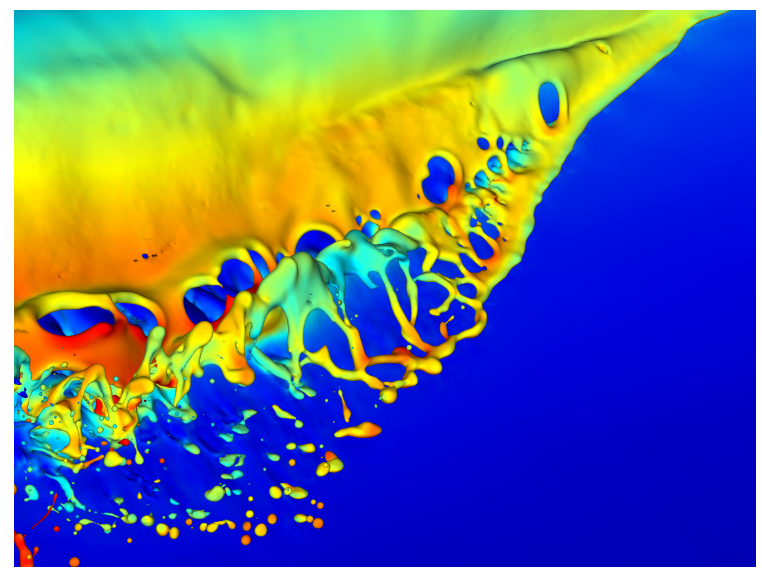

Detail at $t^{\prime}=2.8$

Figure 16. Evolution of the plunging breaker created by the instability of a steep Stokes wave. $0.35<a k<0.45, \mathrm{Re}=40,000, \mathrm{Bo}=33,333$, air/water density and viscosity ratios. Colour represents the magnitude of velocity.

\section{Conclusion}

Current advances in numerical simulation are allowing to go deep in the understanding of the physical processes underlying multi-phase flows. The present work presents three related examples showing the potential of current methods: the investigation of the instabilities in atomization processes, the capillary retraction of a liquid sheet and twoand three-dimensional wave breaking.

The accurate numerical schemes described above together with adaptive mesh refinement techniques have made such simulations possible even for the difficult regimes of air/water systems.

Regarding atomization, a liquid sheet subjected to the effect of a high-velocity jet is considered. The concentration of the mesh cells in the zone near the injector has allowed us to accurately capture the flow structures in this zone whereas further away, the mesh has been coarsened in order to reduce the computational effort. Together with the removal of the small droplets introduced in the system, this has allowed to 


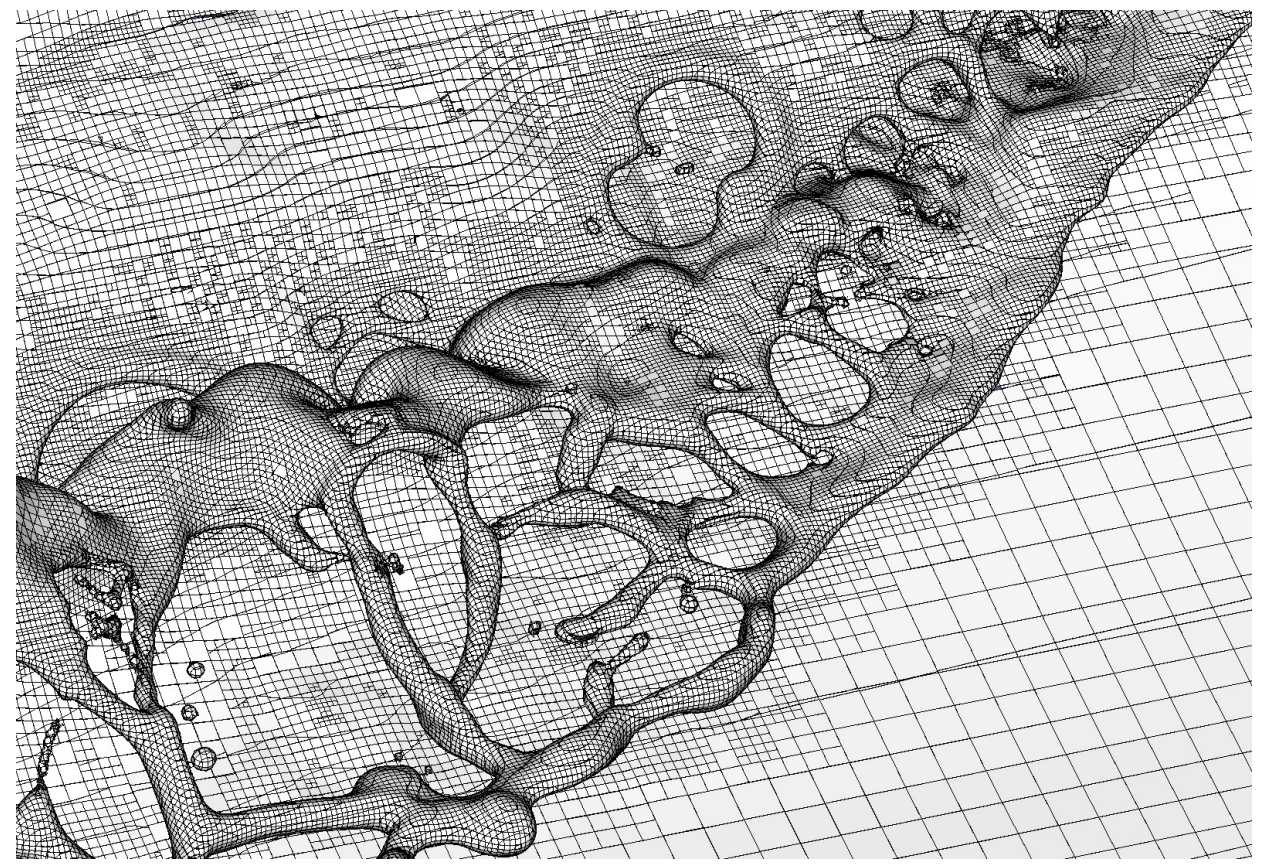

Figure 17. Detail of the adaptive VOF interface representation for the breaking wave at $t^{\prime}=2.8$.

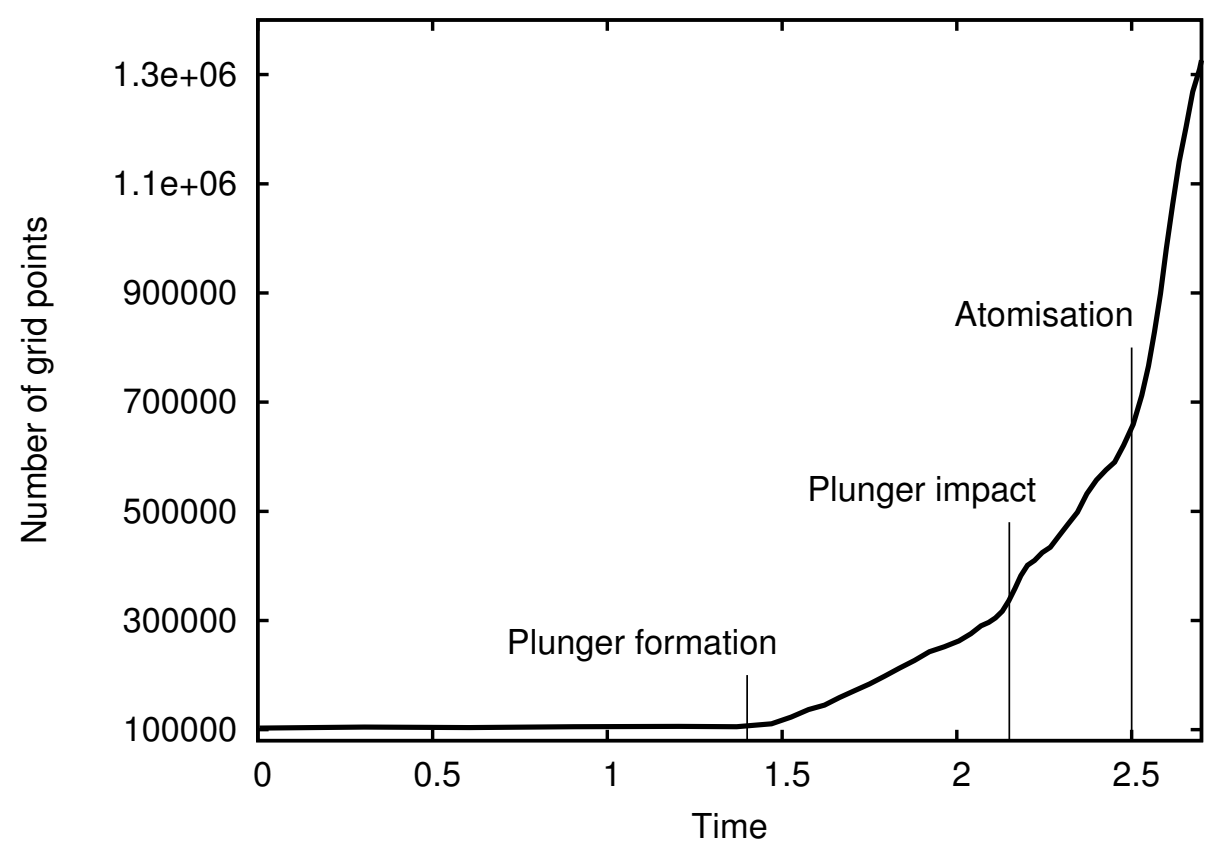

Figure 18. Evolution with time of the total number of grid points for the 3D wave breaking simulation. 
perform accurate simulations in conditions used experimentally to investigate air/water atomization processes. Quantitatively the length of the potential cone correctly fits the experimental measurements. In addition, new mechanisms leading to the appearance of characteristic frequencies have been captured. The new simulations seem to point out that the appareance of the large waves induced into the system are related to the characteristic time required by the flow near the injector to recover its level once the previous wave is propagated downstream. This regime is probably linked to a high momentum ratio $M$ or to high ratios $U_{g} / U_{l}$.

As a second example, the two-dimensional retraction of a water sheet in air is considered. Again, even for large density and viscosity ratios, no numerical instabilities are found. The new simulations have shed some light into the drop-formation process. Two distinct regimes are found for the neck contraction process as a function of the Ohnesorge number. For values larger than 0.016, a smooth neck contraction is observed and its evolution tends to a constant value. For values smaller than 0.01 , a turbulent flow is predicted inside the rim which produces large oscillations in both the neck thickness and the rim shape.

Finally, the preliminary study of air/water wave breaking further illustrates the robustness, accuracy and efficiency of the numerical methods presented. In this case adaptivity is shown to provide a gain of three orders of magnitude in simulation size compared to an equivalent regular-Cartesian-grid simulation. Allowing adaptivity along the interface based on interface curvature reduced the simulation size by a factor of 50 compared to an adaptive technique resolving the interface at constant resolution.

\section{References}

[1] A.S. Almgren, J.B. Bell, P. Colella, L.H. Howell, and M.L. Welcome. A conservative adaptive projection method for the variable density incompressible Navier-Stokes equations. Journal of Computational Physics, 142(1):1-46, 1998.

[2] G. Compère, E. Marchandise, and J.-F. Remacle. Transient adaptivity applied to two-phase incompressible flows. Journal of Computational Physics, 227(3):1923 - 1942, 2008.

[3] M. Dai and D.P. Schmidt. Adaptive tetrahedral meshing in free-surface flow. Journal of Computational Physics, 208(1):228-252, 2005.

[4] G Debrégeas, P de Gennes, and F Brochard-Wyart. The life and death of bare viscous bubbles. Science, Jan 1998.

[5] F. Dias and T.J. Bridges. The numerical computation of freely propagating time-dependent irrotational water waves. Fluid Dynamics Research, 38(12):803-830, 2006.

[6] L. Duchemin, S. Popinet, C. Josserand, and S. Zaleski. Jet formation in bubbles bursting at a free surface. Physics of Fluids, 14:3000, 2002.

[7] J. Eggers. Nonlinear dynamics and breakup of free-surface flows. Reviews of Modern Physics, 69(3):865-930, 1997.

[8] M.M. Francois, S.J. Cummins, E.D. Dendy, D.B. Kothe, J.M. Sicilian, and M.W. Williams. A balanced-force algorithm for continuous and sharp interfacial surface tension models within a volume tracking framework. Journal of Computational Physics, 213(1):141-173, 2006.

[9] D.E. Fyfe, E.S. Oran, and M.J. Fritts. Surface tension and viscosity with Lagrangian hydrodynamics on a triangular mesh. Journal of Computational Physics, 76(2):349-384, 1988. 
[10] D.M. Greaves. A quadtree adaptive method for simulating fluid flows with moving interfaces. Journal of Computational Physics, 194(1):35-56, 2004.

[11] D. Gueyffier, A. Nadim, J. Li, R. Scardovelli, and S. Zaleski. Volume of fluid interface tracking with smoothed surface stress methods for three-dimensional flows. Journal of Computational Physics, 152:423-456, 1998.

[12] M. Herrmann. A balanced force refined level set grid method for two-phase flows on unstructured flow solver grids. Journal of Computational Physics, 227(4):2674-2706, 2008.

[13] M. Kang, R.P. Fedkiw, and X.D. Liu. A boundary condition capturing method for multiphase incompressible flow. Journal of Scientific Computing, 15(3):323-360, 2000.

[14] H. Lamb. Hydrodynamics. Cambridge University Press, 1971.

[15] S. Popinet. Gerris: a tree-based adaptive solver for the incompressible Euler equations in complex geometries. Journal of Computational Physics, 190(2):572-600, 2003.

[16] S. Popinet. The Gerris Flow Solver. http://gfs.sf.net, 2007.

[17] S. Popinet. An accurate adaptive solver for surface-tension-driven interfacial flows. Journal of Computational Physics, 228:5838-5866, 2009.

[18] S. Popinet and S. Zaleski. A front-tracking algorithm for accurate representation of surface tension. International Journal for Numerical Methods in Fluids, 30(6):775-793, 1999.

[19] Y. Renardy and M. Renardy. PROST - A parabolic reconstruction of surface tension for the volume-of-fluid method. Journal of Computational Physics, 183(2):400-421, 2002.

[20] M. Rudman. A volume-tracking method for incompressible multifluid flows with large density variations. International Journal for Numerical Methods in Fluids, 28(2), 1998.

[21] R. Scardovelli and S. Zaleski. Direct numerical simulation of free-surface and interfacial flow. Annual Review of Fluid Mechanics, 31(1):567-603, 1999.

[22] S. Shin, S.I. Abdel-Khalik, V. Daru, and D. Juric. Accurate representation of surface tension using the level contour reconstruction method. Journal of Computational Physics, 203(2):493-516, 2005 .

[23] M. Sussman, P. Smereka, and S. Osher. A level set approach for computing solutions to incompressible two-phase flow. Journal of Computational Physics, 114(1):146-159, 1994.

[24] M. Sussman, K.M. Smith, M.Y. Hussaini, M. Ohta, and R. Zhi-Wei. A sharp interface method for incompressible two-phase flows. Journal of Computational Physics, 221(2):469-505, 2007.

[25] D. J. Torres and J. U. Brackbill. The point-set method: front-tracking without connectivity. J. Comput. Phys., 165:620-644, 2000.

[26] W. Tsai and L. Hung. Three-dimensional modeling of small-scale processes in the upper boundary layer bounded by a dynamic ocean surface. Journal of Geophysical Research, 112(C2), 2007.

[27] W.T. Tsai, S.M. Chen, and C.H. Moeng. A numerical study on the evolution and structure of a stress-driven free-surface turbulent shear flow. Journal of Fluid Mechanics, 545:163-192, 2005.

[28] E. Aulisa, S. Manservisi, R. Scardovelli, and S. Zaleski. Interface reconstruction with least-squares fit and split advection in three-dimensional cartesian geometry. J. Comput. Phys., 225:23012319, 2007.

[29] Anne Bagué, Daniel Fuster, Stéphane Popinet, Ruben Scardovelli, and Stéphane Zaleski. Instability growth rate of two-phase mixing layers from a linear eigenvalue problem and an initial value problem. submitted to Phys. Fluids 2009.

[30] F. Ben Rayana. Étude des instabilités interfaciales liquide-gaz en atomisation assistée et tailles de gouttes. PhD thesis, Institut National Polytechnique de Grenoble, 2007.

[31] M. Brenner and D. Gueyffier. On the bursting of viscous films. Phys. Fluids, 11:737-739, 1999.

[32] A.J. Chorin. On the convergence of discrete approximations to the Navier-Stokes equations. Mathematics of Computation, 23(106):341-353, 1969.

[33] Michel Coantic. Mass transfert across the ocean-air interface : small scale hydrodynamic and aerodynamic mechanisms. PhysicoChemical Hydrodynamics, 1:249-279, 1980.

[34] F. E. C. Culick. Comments on a ruptured soap film. J. Appl. Phys., 31:1128-1129, 1960.

[35] G Debrégeas, P Martin, and F Brochard-Wyart. Viscous bursting of suspended films. Physical 
Review Letters, Jan 1995.

[36] H. Edgerton. Stopping Time. Harry N. Abrams, New York, 1987.

[37] J. Eggers and E. Villermaux. Physics of liquid jets. Rep. Prog. Phys., 71:036601, 2008.

[38] J. M. Fullana and S. Zaleski. Stability of a growing end-rim in a liquid sheet of uniform thickness. Physics of Fluids, 11:952-954, 1999.

[39] D. Fuster, A. Bagué, T. Boeck, L. Le Moyne, A. Leboissetier, S. Popinet, P. Ray, R. Scardovelli, and S. Zaleski. Simulation of primary atomization with an octree adaptive mesh refinement and VOF method. Int. J. Multiphase Flow, 35:550-565, 2009.

[40] C. Josserand and S. Zaleski. Droplet splashing on a thin liquid film. Physics of Fluids, 15:16501657, 2003.

[41] J Keller, A King, and L Ting. Blob formation. Physics of Fluids, Jan 1995.

[42] S. Popinet. Gerris: a tree-based adaptive solver for the incompressible Euler equations in complex geometries. J. Comput. Phys., 190:572-600, 2003.

[43] R. Scardovelli and S. Zaleski. Direct numerical simulation of free-surface and interfacial flow. Annu. Rev. Fluid Mech., 31:567-603, 1999.

[44] M. Song and G. Tryggvason. The formation of thick borders on an initially stationary fluid sheet. Physics of Fluids, 11(9):2487-93, 1999.

[45] G. I. Taylor. The dynamics of thin sheets of fluid III. Disintegration of fluid sheets. Proc. Roy. Soc. London A, 253:313-321, 1959.

[46] A. L. Yarin and D. A. Weiss. Impact of drops on solid surfaces : self-similar capillary waves, and splashing as a new type of kinematic discontinuity. J. Fluid Mech., 283:141-173, 1995. 